\title{
Electron-Beam-Induced Nucleation in an Antisolvent
}

\author{
Tomoya Yamazaki $^{1 *}$ and Yuki Kimura ${ }^{1}$ \\ 1. Institute of Low Temperature Science, Hokkaido University, Sapporo, Japan \\ * Corresponding author: yamazaki@lowtem.hokudai.ac.jp
}

The crystallization from a solution is an important process in many systems, such as the creation of nanomaterials, the crystallization of proteins for molecular structure analysis, the production of minerals from living organisms, etc. In order to understand its physical picture, in situ observation using a variety of microscopies is a powerful method for visualizing such process directly. However, the understanding of early stages of crystallization, especially appearance of crystal nuclei (nucleation) and subsequent crystal growth under sub-micrometer scale, is still limited because of limitation of techniques to observe these rapid, unexpected and small-scale phenomena in real time. Liquid cell transmission electron microscopy (LC-TEM) is a promising method for observing the early stages of crystallization directly because the spatial resolution of this method is in nanometer scale [1]. In fact, several groups succeeded in observing the nucleation of calcium carbonate [2] and protein [3] crystals using LC-TEM, and these observations suggest that the pathways of the nucleation are multiple, and the nucleation of some crystalline phases are multistep. However, behind the mechanisms in the selectivity of the pathways and steps of nucleation are still incompletely understood. One of the reasons of this problem is great difficulty of capturing a moment of a nucleation event because the observable area of this method is small $\left(<100 \mu \mathrm{m}^{2}\right)$ and the event is typically very rare in the tiny volume within the area. In order to observe nucleation events efficiently, a supersaturation of a sample solution should be increased with observing the solution. To make the situation, we focus on using the anti-solvent crystallization technique, which crystallize a solute to add a solvent with extremely low solubility of the solute (antisolvent), to LC-TEM. Here we report our attempts to observe the nucleation of salt crystals using their anti-solvent by LC-TEM.

We used a TEM with a field-emission gun at an acceleration voltage of $200 \mathrm{keV}$ (JEOL JEM-2100F) and the TEM holder (Poseidon 300, Protochips Inc.) which provides the observation of a liquid sample using liquid cells of silicon chips with thin silicon nitride windows. We enclosed an aqueous solution of sodium chlorate under equilibrium at room temperature in the liquid cell. The TEM holder has two ports for injection of liquid, and one of the ports was filled by acetone which is the anti-solvent of sodium chlorate. After assembling the liquid cell in the TEM holder, we started monitoring the sample solution. Then the filled acetone was put into the liquid cell by syringe pump to mix with the aqueous solution.

Before and immediately after mixing the two solutions, any crystals could not be observed. However, after a short time, the dendritic crystal (Fig. 1) appeared only in a field of view. If we moved the field of view to an area with no crystal, a dendritic crystal appeared within a few seconds again. These observations suggest that the electron-beam-induced nucleation occurred. The analyses of nucleated crystals and their nucleation mechanisms are progressing.

In summary, we propose the method for observing the nucleation by LC-TEM easily using the salt and its anti-solvent. The trigger of the nucleation is irradiation of electron beam although the detailed mechanisms are still veiled [4]. 


\section{References:}

[1] N de Jonge and FM Ross, Nat. Nanotechnol. 6 (2011), p. 695.

[2] MH Nielsen et al., Science 345 (2014), p. 1158.

[3] T Yamazaki et al., Proc. Natl. Acad. Sci. 114 (2017), p. 2154.

[4] This work was supported partly by Grant for Basic Science Research Projects from the Sumitomo Foundation (Grant Number 170738) and JSPS KAKENHI Grant Numbers JP15H05731 and JP18J01470. TY is grateful for the Research Fellowship of Japan Society for the Promotion of Science for Young Scientists.
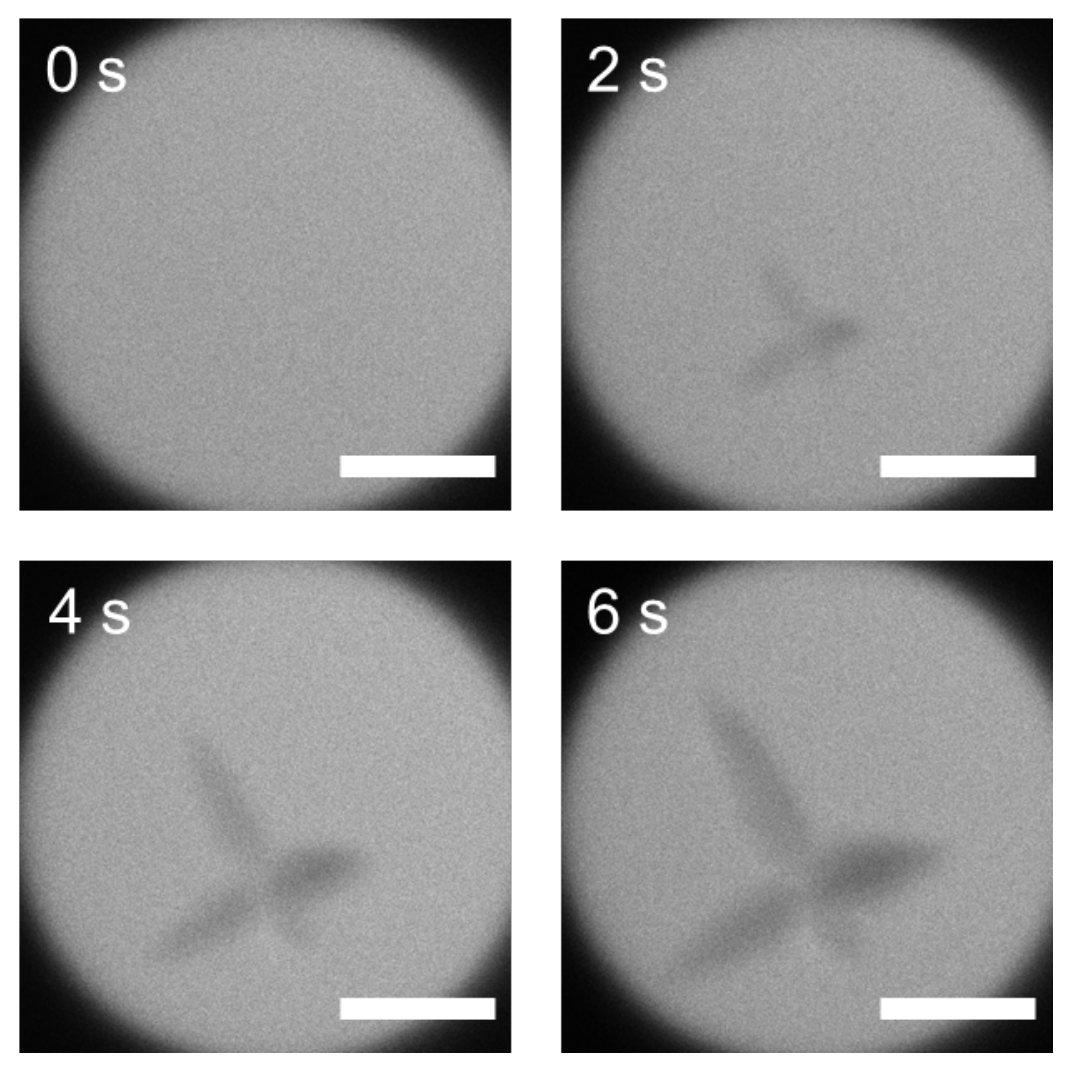

Figure 1. Bright-field TEM images of a crystallization process observed in this system. Immediately before the onset of the nucleation event (at $0 \mathrm{~s}$ ), there is nothing in the filed of view. Then the crystal appeared and grew continuously. The scale bars are $1 \mu \mathrm{m}$. 